\title{
A study on the existence of null labelling for 3-hypergraphs
}

\author{
N. Di Marco ${ }^{1}$, A.Frosini ${ }^{1}$, and W.L. Kocay ${ }^{2}$ \\ 1 Dipartimento di Matematica e Informatica, Università di Firenze, \\ Firenze, Italy, \\ \{niccolo.dimarco, andrea.frosini\}@unifi.it \\ 2 Department of Computer Science and St. Pauls College, University of Manitoba, \\ Winnipeg, Manitoba, CANADA
}

\begin{abstract}
A 3-uniform hypergraph $H$ consists of a set $V$ of vertices, and $E \subseteq\left(\begin{array}{l}V \\ 3\end{array}\right)$ triples. Let a null labelling be an assignment of \pm 1 to the triples such that each vertex has signed degree equal to zero. Assumed as necessary condition the degree of every vertex of $H$ to be even, the Null Labelling Problem consists in determining whether $H$ has a null labelling. Although the problem is NP-complete, the subclasses where the problem turns out to be polynomially solvable are of interest. In this study we define the notion of 2-intersection graph related to a 3 -uniform hypergraph, and we prove that the existence of a Hamiltonian cycle there, is sufficient to obtain a null labelling in the related hypergraph. The proof we propose provides an efficient way of computing the null labelling.
\end{abstract}

Keywords: discrete tomography, 3-hypergraph, null labelling, hamiltonian cycle

AMS classification: 05C65, 05C22, 05C99

\section{Introduction}

The characterization of simple graphs from their degree sequences has been a challenging problem whose solution dates back to the well known result of Erdös and Gallai [7] in 1960: an integer sequence $d=\left(d_{1}, \ldots, d_{n}\right)$ is graphic if and only if $\sum_{i=1}^{n} d_{i}$ is even and

$$
\sum_{i=1}^{k} d_{i} \leq k(k-1)+\sum_{i=k+1}^{n} \min \left\{k, d_{i}\right\}, \quad 1 \leq k \leq n .
$$

This same problem related to hypergraphs remained open until 2018 when Deza et al. proved its NP-completeness [6] even in the simplest case of 3-uniform hypergraphs.

This paper is eligible for the best student paper award. 
Before this result many necessary and a few sufficient conditions are present in the literature, and they mainly rely on a result by Dewdney [5]. As an example, Behrens et al. [1] proposed a sufficient and polynomially testable condition for a degree sequence to be $k$-graphic; their result still does not provide any information about the associated $k$-hypergraphs. Soon after, in $[3,9,10]$, a series of polynomial time algorithms was proposed to reconstruct one of the $k$ hypergraphs associated to each degree sequence of some classes including that studied in [1]. These results use tools borrowed from discrete tomography (DT), a young discipline concerned with inverse problems. In particular, it deals with the determination of geometrical properties of unknown objects, usually modelled by binary matrices, from their projections, i.e., a quantitative measurements of the number of primary constituents along prescribed directions (see $[12,13]$ for the main results and the open problems). So, the reconstruction of a $k$-hypergraph from its degree sequence can be translated in the DT framework as the reconstruction of a binary matrix, i.e., its incidence matrix, from horizontal and vertical projections, i.e., the constant vector of entries $k$ and the degree sequence, respectively.

However, some relevant related questions remain open, in particular the study of the uniqueness (up to isomorphism) of $k$-hypergraphs sharing the same degree sequence. In our study, we focus on this problem by considering two hypergraphs $H_{1}$ and $H_{2}$ with the same degree sequence. Their symmetric difference $H_{1} \ominus H_{2}$ produces a null hypergraph, when assigning a +1 label to each hyperedge of $H_{1}$ and $H_{2}$. Vice versa, given a null hypergraph $H$, call $H_{1}$ the hypergraph with the same vertex as $H$ but only the positive hyperedges of $H$ and $H_{2}$ the same but with only the negative hyperedges of $H$. It's easy to see that $H_{1}$ and $H_{2}$ have the same degree sequence.

In [14], the notion of null hypergraph has been used to study the changes of hyperedges that allow one to move through all the 3-hypergraphs with the same degree sequence. The present research links the null label of a $k$-hypergraph with its 2-intersection graph, showing that the existence of a Hamiltonian cycle in the 2 -intersection graph is sufficient to define a null label of the related $k$-hypergraph.

In Section 2, we recall the basic definition of graphs and hypergraphs, then we introduce some useful notation used throughout the paper, together with the notion of 2-intersection graph. Some results about null hypergraphs are also provided. In Section 3, we prove some properties of the 2-intersection graph of a $k$-hypergraph. Finally, we define a null labelling of a 3-hypergraph from a given Hamiltonian cycle of its 2-intersection graph.

\section{Definitions and known results}

A graph $G$ is defined as a pair $G=(V, E)$ such that $V=\left\{v_{1}, \ldots, v_{n}\right\}$ is the set of vertices and $E$ is a collection of pairs of vertices called edges.

The notion of graph can be generalized to that of hypergraph by removing the constraint on the cardinality of the edges: $H=(V, E)$ is a hypergraph with $V=\left\{v_{1}, \ldots, v_{n}\right\}$ the set of vertices and $E$ a collection of subsets of vertices 
called hyperedges. We choose to abbreviate the vertex notation $v_{i}$ with its only index $i$, when no misunderstandings may arise.

In the sequel, we will consider only graphs and hypergraphs that are simple, i.e., they do not allow singleton (hyper)edges or (hyper)edges that are contained in or equal to other edges. The degree of a vertex $v \in V$ is the number of (hyper)edges containing $v$. The degree sequence $\left(d_{1}, d_{2}, \ldots, d_{n}\right)$ of a (hyper)graph is the list of its vertex degrees usually arranged in non-increasing order. A hypergraph whose hyperedges have fixed cardinality is called $k$-uniform, or simply a $k$-hypergraph, and its degree sequence is called a $k$-graphic sequence. Note that the case $k=2$ corresponds to graphs, and a 2-graphic sequence is called graphic. Our study will focus on 3-hypergraphs.

Given a (hyper)graph, we can assign a labelling $l$ with labels +1 or -1 to each (hyper)edge, resulting in positive and negative (hyper)edges. The positive degree of a vertex $v$ is $d_{l}^{+}(v)$, the number of positive (hyper)edges containing $v$. The negative degree is $d_{l}^{-}(v)$, the number of negative (hyper)edges containing $v$. The signed degree of each vertex $v$ is $d_{l}(v)=d_{l}^{+}(v)-d_{l}^{-}(v)$. The unsigned degree is $\operatorname{deg}(v)=d_{l}^{+}(v)+d_{l}^{-}(v)$. The subscript indicating the labelling will be omitted when no misunderstandings may arise.

An assignment of \pm 1 to the (hyper)edges of a (hyper)graph is a null labelling if $d(v)=0$, for all vertices $v$. A (hyper)graph with a null labelling is said to be a null (hyper)graph. An obvious necessary condition for a (hyper)graph to have a null labelling is that each vertex must have even degree, i.e., it is an even (hyper)graph.

The following lemma characterizes graphs with a null labelling.

Lemma 1. A graph $G$ has a null labelling if and only if every connected component is an Eulerian graph with an even number of edges.

Proof. If a graph has a null labelling, then it must has an even number of edges. Therefore, the necessity follows from the comments preceding the lemma. To prove sufficiency, consider a connected component which is an Eulerian graph with an even number of edges. By following an Euler tour, assigning alternately \pm 1 to alternate edges, a null labelling is obtained.

This lemma also characterizes the graphs with even degrees and an even number of edges that do not have a null labelling: they must be disconnected graphs such that at least two connected components have an odd number of edges. The smallest graph with a null labelling is a cycle on four vertices, which we denote by $C_{4}$.

Moving to hypergraphs the situation becomes more complex. Let $H_{1}$ and $H_{2}$ be two hypergraphs with the same vertex set, and the same degree sequence $\left(d_{1}, d_{2}, \ldots, d_{n}\right)$. Assign +1 to the triples of $H_{1}$ and -1 to the triples of $H_{2}$, and construct $H_{1} \ominus H_{2}$. It is a hypergraph with a null labelling. This raises the question of whether there is a characterization of null hypergraphs. In [8] it is shown that the problem of finding a null labelling even for the simplest case of 3-hypergraphs is NP-complete. 
Hypergraph Null Labelling Problem: Let $H$ be a connected, even 3-hypergraph. When can \pm 1 be assigned to the hyperedges of $H$ to produce a null-labelled 3hypergraph?

The intersection graph of a 3-hypergraph $H$ is denoted $I(H)$. Its vertices are the hyperedges of $H$. Two hyperedges are adjacent if their intersection is non-empty. This is an extension of the idea of a line graph to 3-hypergraphs. In [8] the following result was proved.

Theorem 1. Let $H$ be a connected, even 3-hypergraph, in which every vertex has degree two. Then $H$ has a null labelling if and only if $I(H)$ is bipartite.

However, the inspection of the intersection graph does not provide evidence, in general, of the existence of a null labelling in the related 3-hypergraph as shown in the following example.

Example 1. Consider the following 3-hypergraphs $H_{1}$ and $H_{2}$ on six vertices and whose hyperedges, arranged in matrix form, are:

$$
H_{1}=\left[\begin{array}{lll}
1 & 2 & 3 \\
1 & 4 & 5 \\
2 & 4 & 6 \\
3 & 5 & 6
\end{array}\right] \quad H_{2}=\left[\begin{array}{lll}
1 & 2 & 5 \\
2 & 3 & 5 \\
2 & 3 & 4 \\
1 & 2 & 4
\end{array}\right]
$$

It is easy to check that the vector of labels $l=(1,-1,1,-1)$, where $l(i)$ is the label of the $i$-th hyperedge of the 3-hypergraph or, equivalently, of the $i$-th row in the matrix arrangement of its hyperedges, is a null label for $H_{2}$, while $H_{1}$ has no null labelling. However, $H_{1}$ and $H_{2}$ have the same intersection graph $K_{4}$, i.e. the complete graph on four vertices.

Relying on this fact, we modify the notion of intersection graph as follows: the 2-intersection graph of a 3-hypergraph $H$ is denoted $I_{2}(H)=\left(V_{2 H}, E_{2 H}\right)$. Its vertices $V_{2 H}=\left\{v_{e_{1}}, \ldots, v_{e_{m}}\right\}$ represents the hyperedges $E=\left\{e_{1}, \ldots, e_{m}\right\}$ of $H$. Two hyperedges are adjacent, i.e., they belong to the same edge $\left\{v_{e_{i}}, v_{e_{j}}\right\} \in E_{2 H}$, if $e_{i}$ and $e_{j}$ share a pair of vertices of $H$ (see Example 2 and the related Fig. 2). In the sequel, we label the edge $\left\{v_{e_{i}}, v_{e_{j}}\right\} \in E_{2 H}$ with the pair of vertices that are shared by $e_{i}$ and $e_{j}$, if needed.

\section{Hypergraph and 2-intersection graph}

Let $H=(V, E)$ be a 3-uniform hypergraph (3-hypergraph). We study some properties of the 2-intersection graph that are relevant to obtain information about the existence of a null labelling in the related 3-hypergraph. In particular, we will consider Hamiltonian cycles, as they appear to be relevant to null labellings.

Question: Consider the 2-intersection graph $G$ of a connected, even 3-hypergraph $H$. Is it possible to construct a null-label from a Hamiltonian cycle in $G$ ? We want to prove that if $G$ is Hamiltonian then $H$ has a null-label. 
We know that a connected, even graph $G$ is Eulerian. Its line-graph $L(G)$ is the intersection graph of its edges. An Euler tour in $G$ corresponds to a Hamiltonian cycle in $L(G)$, and conversely. And it also corresponds to a null labelling of $G$. Thus, Hamiltonian cycles in $L(G)$ can be used to determine null labellings of $G$.

The 2-intersection graph of a 3-hypergraph $H$ is similar to the line graph of a graph. It is well known (see Harary [11]) that a line graph has a unique decomposition into maximal cliques, with at most a vertex in common. Thus we consider cliques in 2-intersection graphs $I_{2}(H)$ of 3-hypergraphs.

Property 1. Let $H=(V, E)$ be a 3-hypergraph. The hyperedges $e_{1}, \ldots, e_{n} \in E$ sharing the same pair of elements form a clique in the 2-incidence graph $I_{2}(H)$.

This property is a direct consequence of the definition of 2-incidence graph. There is another kind of clique in a 2-intersection graph. Define a triangle in a 3 -hypergraph $H$ to be three hyperedges of the form $\{1,2,3\},\{2,3,4\},\{3,4,1\}$. Any two of them intersect in a pair, so they also form a triangle in $I_{2}(H)$.

Property 2. Let $H=(V, E)$ be a 3-hypergraph. There are two kinds of cliques in $I_{2}(H)$, namely those hyperedges all sharing a common pair, and those hyperedges deriving from a triangle in $H$.

Example 2. Consider the following hypergraph $H=(V, E)$ in which $V=$ $\{1, \ldots, 6\}$ and $E=\{\{3,4,5\},\{3,5,6\},\{1,3,5\},\{3,4,6\},\{2,4,6\},\{4,5,6\}$, $\{1,2,6\},\{2,3,5\},\{1,2,5\},\{1,2,4\},\{1,2,3\},\{1,4,6\}\}$.

The 2-intersection graph of $H$ is shown in Figure 1. According to Property 1, one can check that the edges with the same label form a clique.

Our purpose here aims at detecting a strategy that starting from a Hamiltonian cycle $\mathrm{C}$ of $I_{2}(H)$ allows one to define a labelling of its vertices that is a null labelling of $H$.

A first naive strategy consists in alternately labelling \pm 1 the vertices of $C$ to obtain a null labelling of $H$. This is a strategy that works for line graphs of even graphs. Let $v_{e_{i}}$ and $v_{e_{j}}$ be two consecutive elements of $C$, with $e_{i}=\{u, x, y\}$ and $e_{j}=\{v, x, y\}$ hyperedges of $H$, we note that such a labelling maintains the signed degree of $x$ and $y$. On the other hand, it increases/decreases by one the signed degree of $u$ and $v$. So, we see that the alternating labelling of $C$ does not always provide a null labelling of $H$, as witnessed by the following example.

Example 3. Consider the 3-hypergraph $H=(V, E)$ on six vertices and $E=$ $\left\{e_{1}, \ldots, e_{8}\right\}$, where $e_{1}=\{1,2,3\}, e_{2}=\{1,2,4\}, e_{3}=\{1,2,5\}, e_{4}=\{1,2,6\}, e_{5}=$ $\{1,3,4\}, e_{6}=\{1,3,5\}, e_{7}=\{2,3,5\}, e_{8}=\{2,5,6\}$.

The related 2-intersection graph $I_{2}(H)$ in Fig. 2 has the Hamiltonian cycle $C_{1}=\left(v_{e_{1}}, v_{e_{3}}, v_{e_{2}}, v_{e_{4}}, v_{e_{8}}, v_{e_{7}}, v_{e_{6}}, v_{e_{5}}, v_{e_{1}}\right)$

It is easy to check that alternately labelling \pm 1 the vertices of $C_{1}$, starting with +1 , we obtain the null labelling $l_{1}=(1,1,-1,-1,-1,1,-1,1)$ on the eight hyperedges of $H$ such that $l_{1}(i)$ is the label of $e_{i}$, with $1 \leq i \leq 8$. 


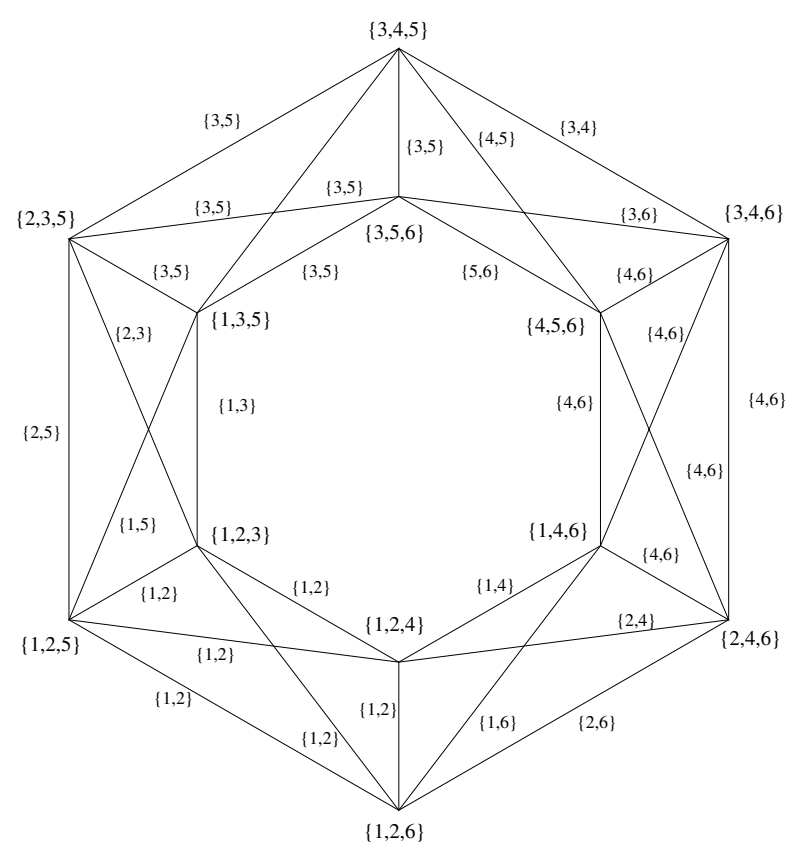

Fig. 1. The 2-intersection graph of the 3-hypergraph $H$ in Example 2. The edges are labelled according to the pairs shared by their vertices.

Unfortunately, not every Hamiltonian cycle provides a null labelling. A second Hamiltonian cycle $C_{2}=\left(v_{e_{1}}, v_{e_{2}}, v_{e_{3}}, v_{e_{4}}, v_{e_{8}}, v_{e_{7}}, v_{e_{6}}, v_{e_{5}}, v_{e_{1}}\right)$ exists such that the alternating labelling $l_{2}=(1,-1,1,-1,-1,1,-1,1)$ is not null on $H$, as $d\left(v_{4}\right)=-2$ and $d\left(v_{5}\right)=+2$.

Let us introduce some notation: suppose that $v_{e_{i}}$ and $v_{e_{j}}$ are two consecutive vertices of the Hamiltonian cycle $C$ of $I_{2}(H)$, with $e_{i}=\{u, x, y\}$ and $e_{j}=$ $\{v, x, y\}$. We say, by extension, that $v_{e_{j}}$ contains the node $v \in H$. We see that $v \notin e_{i}$. There may be several consecutive vertices of $C$ that contain $v$. Denote by $p_{v}=\left(v_{e_{j_{1}}}, \ldots, v_{e_{j_{k}}}\right)$ the longest sub-path of $C$ starting in $v_{e_{j}}$ such that every vertex of $p_{v}$ contains $v$. Let $l\left(p_{v}\right)$ denote the labels of the vertices of $p_{v}$, and let $\sigma\left(l\left(p_{v}\right)\right)$ denote the sum of the elements of $l\left(p_{v}\right)$, and let $\left|p_{v}\right|$ denote the length $k-1$ of $p_{v}$, i.e., its number of edges. In the case when $p_{v}$ contains just a single vertex, $\left|p_{v}\right|=0$.

In this example, $e_{i}=\{u, x, y\}$ is clearly the last vertex in a path $p_{u}$. Therefore we define $\operatorname{next}\left(p_{u}\right)=p_{v}$, for this $p_{u}$ and $p_{v}$, i.e., given a path $p_{u}, \operatorname{next}\left(p_{u}\right)$ is the path beginning at the first vertex following the last vertex of $p_{u}$. In general, $C$ may contain several different sub-paths of the form $p_{v}$, for each vertex $v$; we indicate them by $p_{v}^{1}, \ldots, p_{v}^{n}$. An example is shown in Figure 3.

Property 3. Given an alternating labelling \pm 1 on the vertices of a Hamiltonian cycle $C$ of $I_{2}(H)$. For each sub-path $p_{v}=\left(v_{e_{j_{1}}}, \ldots, v_{e_{j_{k}}}\right)$, the following holds: 


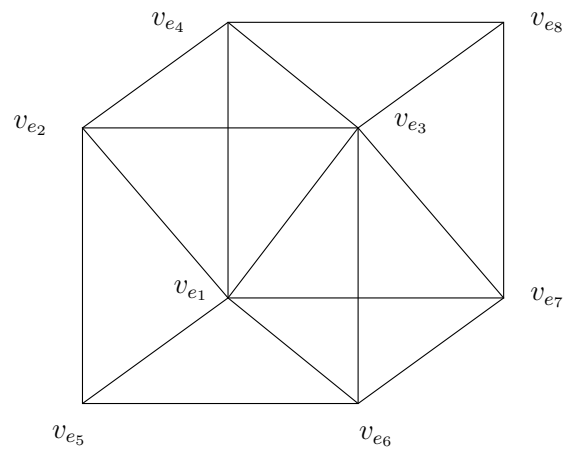

Fig. 2. The 2-intersection graph of the 3-hypergraph considered in Example 3.

- if $p_{v}$ has odd length, then $\sigma\left(l\left(p_{v}\right)\right)=0$, so that the labels of the hyperedges $e_{j_{1}}, \ldots, e_{j_{k}}$ containing $v$ sum to zero in $H$. In this case the first and the last vertex of $p_{v}$ have different labels;

- if $p_{v}$ has even length, then $\sigma\left(l\left(p_{v}\right)\right) \neq 0$ and the sum of the labels of the hyperedges $e_{j_{1}}, \ldots, e_{j_{k}}$ containing $v$ contribute +1 or -1 to the signed degree of $v$. In this case, the extremal vertices of $p_{v}$ have the same label.

The proof of this property is straightforward. Figure 3 shows the 2-intersection graph of a 3-hypergraph on six vertices and eight hyperedges. One of its Hamiltonian cycles and the sub-paths related to the vertices of $H$ are highlighted. Note that the paths in the diagram circle around the right edge of the diagram back to the left edge.

Let us continue analyzing the properties of the alternating labelling $l(C)$ of a Hamiltonian cycle $C$ of $I_{2}(H)$. Property 3 assures that, if the labelling $l(C)$ produces a signed degree $d_{l}(v)=d \neq 0$ for vertex $v$, then there exists at least $p_{v}^{1}, \ldots, p_{v}^{|d|}$ subpaths with the same sum of labels. An example is seen in Figure 3.

We define the distance between two paths $p_{u}$ and $p_{v}$ as the distance along $C$ between the last point of $p_{u}$ and the first point $p_{v}$. We observe that any two of the previous $|d|$ subpaths have even distance. The above observations lead to the following lemmas

Lemma 2. Let $H$ be an even 3-hypergraph and $I_{2}(H)$ its 2-intersection graph. If $I_{2}(H)$ has a Hamiltonian cycle $C$, an alternating \pm 1 labelling $l(C)$ defines a null label of $H$ if and only if, for each $v \in V$ :

i) each subpath $p_{v}$ has odd length; OR

ii) the number of subpaths of $v$ having even length is even and the sum of their labels is zero.

We emphasize that $i$ ) expresses the condition that, for each vertex $v$ of $H$, there are the same number of subpaths of $p_{v}$ having label +1 as -1 .

We also have the following lemma. 

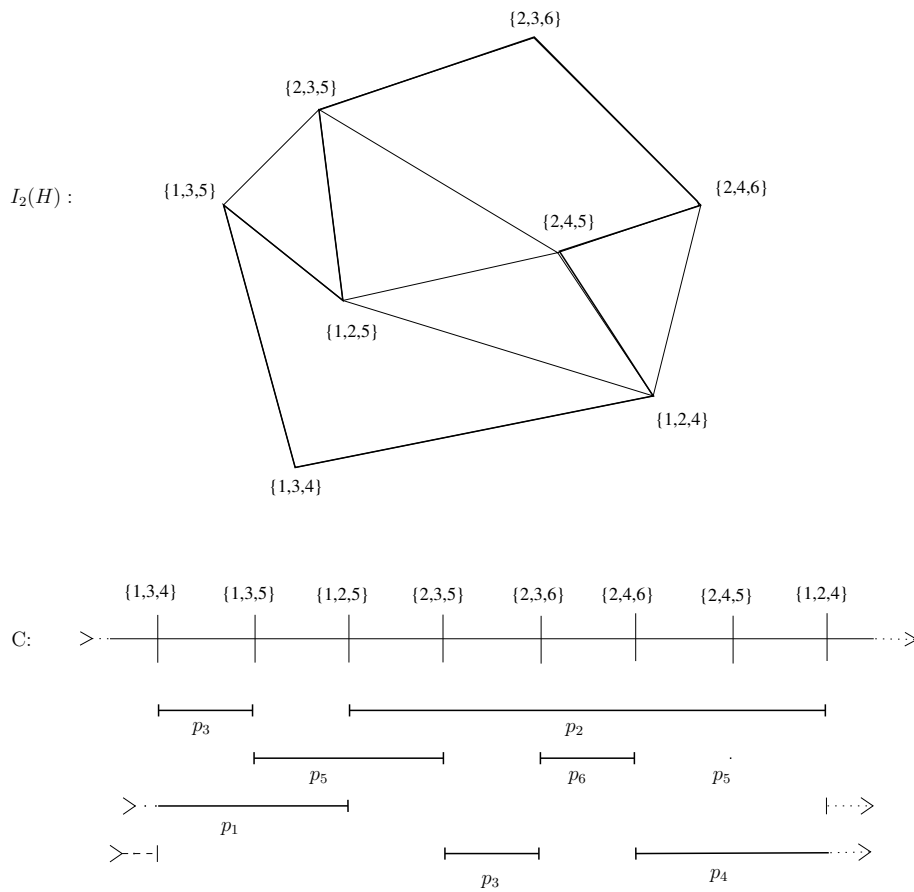

Fig. 3. A 2-intersection graph $I_{2}(H)$ and one of its Hamiltonian cycles $C$ are shown. The sub-paths $p_{v}$ related to the vertices of $H$ are highlighted. Note that in each vertex of $I_{2}(H)$ starts and ends two sub-paths related to two (non necessarily distinct) vertices of $H$.

Lemma 3. Let $H$ be a 3-hypergraph and $C$ a Hamiltonian cycle of $I_{2}(H)$, and let $v_{e}=\{u, x, y\} \in V_{2 H}$ be a vertex of $I_{2}(H)$. There are exactly three subpaths containing $v_{e}$, namely $p_{u}, p_{x}$, and $p_{y}$. One of them begins at $v_{e}$ and one of them ends at $v_{e}$ (possibly the same path begins and ends at $v_{e}$ ).

Proof. The next vertex of $C$ intersects $v_{e}$ in two vertices, say $x$ and $y$. Then $e^{\prime}=\{v, x, y\}$ is the next vertex of $C$. The previous vertex also intersects $v_{e}$ in two vertices, wlog, either $x, y$ or $u, x$. Let $e^{\prime \prime}$ be the previous vertex of $C$. Then either $e^{\prime \prime}=\{w, x, y\}$ or $e^{\prime \prime}=\{u, x, w\}$, for some $w$. In the first case, we have $p_{u}$ begins and ends at $v_{e}$. In the second case, we have $p_{u}$ ends at $v_{e}$ and $p_{y}$ begins.

In the sequel, we describe an algorithm that modifies an alternating \pm 1 labelling of a Hamiltonian cycle $C$ not satisfying the conditions of Lemma 2 in order to obtain a null labelling of $H$. This algorithm relies on the $S_{w i t c h}()$ operator defined as follows: given two even sub-paths $p_{u}=\left(v_{e_{i_{1}}}, \ldots, v_{e_{i_{k}}}\right)$ and $p_{v}=$ $\left(v_{e_{j_{1}}}, \ldots, v_{e_{j_{k^{\prime}}}}\right)$, where $p_{v}=\operatorname{next}\left(p_{u}\right)$, and $e_{i_{k}} \neq e_{j_{1}}$, the operator $S_{w i t c h}\left(p_{u}, p_{v}\right)$ produces a new labelling $l^{\prime}(C)$ by changing the signs of $e_{i_{k}}$ and $e_{j_{1}}: l^{\prime}\left(e_{i_{k}}\right)=$ 
$-l\left(e_{i_{k}}\right)$ and $l^{\prime}\left(e_{j_{1}}\right)=-l\left(e_{j_{1}}\right)$; and keeping the remaining labels of $l(C)$ unchanged. Figure 4 shows an example of the action of $\operatorname{Switch}\left(p_{2}, p_{5}\right)$.

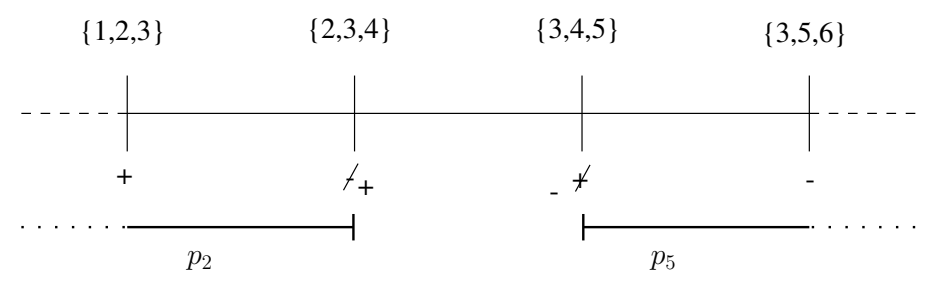

Fig. 4. Example of $\operatorname{Switch}\left(p_{2}, p_{5}\right)$ between the two consecutive paths $p_{2}$ and $p_{5}$, i.e., such that $p_{5}=\operatorname{next}\left(p_{2}\right)$.

We will start with an alternating labelling $l(C)$, and gradually change it using Switch().

Property 4. Let $H$ be a 3-hypergraph, $C$ a Hamiltonian cycle of $I_{2}(H)$, and $l$ a \pm 1 labelling of $C$. Consider a sub-path $p_{u}$ of $C$ whose last element $v_{e_{i}}$ with label +1 , and the sub-path $p_{v}=\operatorname{next}\left(p_{u}\right)$ whose first element $v_{e_{j}}$ with label -1 . The operator $\operatorname{Switch}\left(p_{u}, p_{v}\right)$ modifies $l$ into $l^{\prime}$ so that $d_{l^{\prime}}(u)=d_{l}(u)-2$, $d_{l^{\prime}}(v)=d_{l}(v)+2$ and all the remaining signed degrees are left unchanged.

Proof. Without loss of generality, assume that $e_{i}=\{u, x, y\}$ and $e_{j}=\{v, x, y\}$. It is immediate that the change of the opposite labels of $e_{i}$ and $e_{j}$ keeps the signed degrees of $x$ and $y$, while it subtracts 2 from $u$ and adds 2 to $v$. As the starting labels of $e_{i}$ and $e_{j}$ are opposite, a symmetric result holds.

The algorithm Balance() defined below modifies a labelling $l(C)$ of a Hamiltonian cycle $C$ of $I_{2}(H)$ in order to change, after a sequence of successive applications of the $S$ witch() operator, the signed degree of two input vertices $u$ and $v$ of $H$, if possible, otherwise it gives failure.

First, we prove that the the algorithm Balance() computes a null labelling starting from the alternating labelling $l(C)$ in the easiest case of having only two signed degrees $u$ and $v$ different from zero, in particular +2 and -2 , respectively.

Lemma 4. Let $H$ be a 3-hypergraph, $C$ a Hamiltonian cycle of $I_{2}(H)$, and $l$ an alternating labelling of $C$. If $u$ and $v$ are the only nodes of $H$ with signed degree different from zero, in particular $d_{l}(u)=+2$ and $d_{l}(v)=-2$, then Balance $(u, v, l(C))$ returns a null labelling $l^{\prime}(C)$ of $H$.

Proof. Since $d_{l}(u)=2$, there exists at least one subpath $p_{u}$ such that $\left|p_{u}\right|$ is even and $\sigma\left(l\left(p_{u}\right)\right)=+1$, i.e. it starts and ends with two elements labelled with +1 .

The While cycle starts by performing the switch between $p_{u}$ and $p_{j}=$ next $\left(p_{u}\right)$ and $l$ is updated to $l^{\prime}$. Since $l$ is an alternating labelling, the first element 


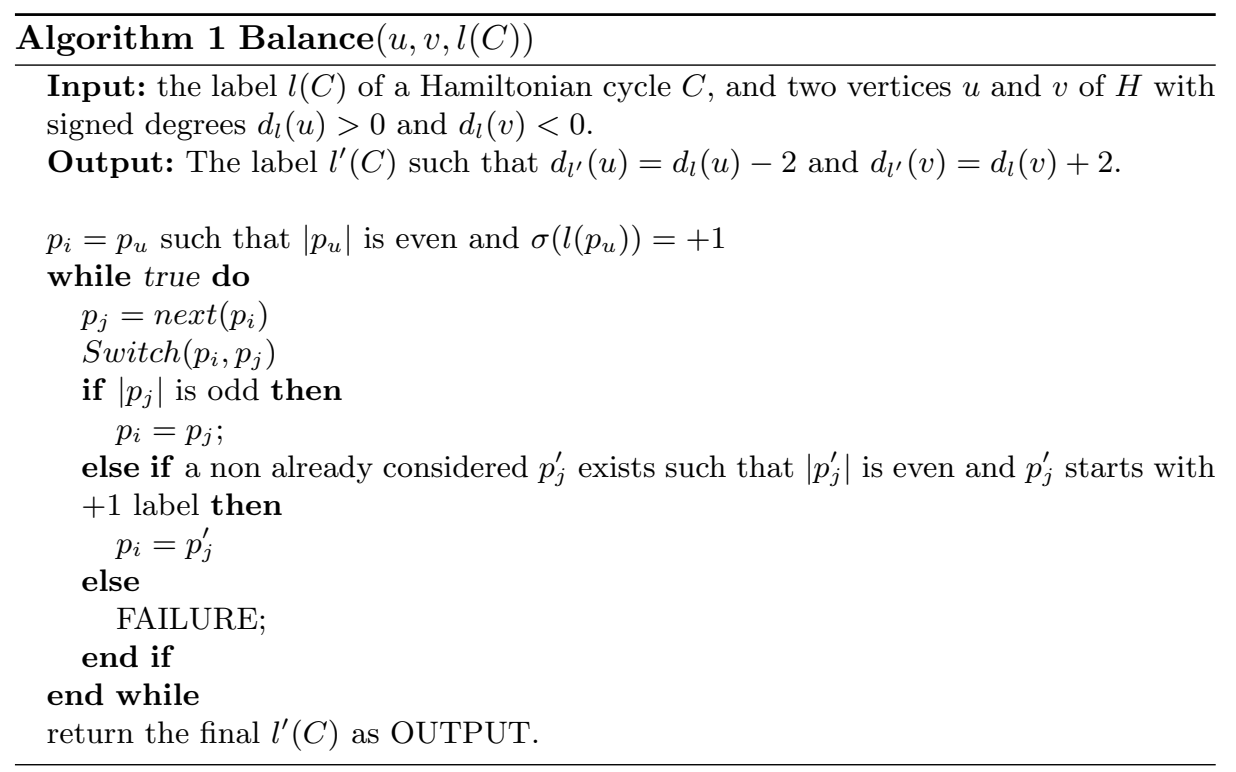

of $p_{j}$ has label -1, so after $\operatorname{Switch}\left(p_{u}, p_{j}\right)$, we have $d_{l^{\prime}}(u)=0, d_{l^{\prime}}(j)=d_{l}(j)+2$, and by Property 4 , the other signed degrees do not change. Now, if $\left|p_{j}\right|$ is even and $j=v$, then $d_{l^{\prime}}\left(p_{v}\right)=0$ and $l^{\prime}$ is the desired null labelling.

On the other hand, if $\left|p_{j}\right|$ is odd, then it ends with a +1 label and its successor next $\left(p_{j}\right)$ starts with a -1 . So, after updating $p_{i}=p_{j}$ and $p_{j}=\operatorname{next}\left(p_{i}\right)$, Switch $\left(p_{i}, p_{j}\right)$ is again performed and the new $l^{\prime}$ changes back the signed degree of $i$ to zero, while +2 is added to the signed degree of the new $j$. Note that in this case, even if $j=v$, we decide to continue with the algorithm until reaching a $\left|p_{v}\right|$ of even length and such that $\sigma\left(l^{\prime}\left(p_{v}\right)\right)=-1$.

If the last case $\left|p_{j}\right|$ is even and $j \neq v$ occurs, then we move to another new even $p_{j}^{\prime}$ such that $\sigma\left(l\left(p_{j}^{\prime}\right)\right)=+1$. Such a $p_{j}^{\prime}$ always exists since $d_{l}(j)=0$, and consequently the number of even sub-paths containing $j$ whose labels sum up to +1 equals those whose labels sum up to -1 . So, FAILURE never occurs starting from an alternating labelling $l$.

Finally, the result is obtained by observing that Balance $(u, v, l(C))$ does not loop since the number of subpath of $C$ is finite and each of them is involved in the while cycle at most once since the procedure always switches a sub-path that ends with $\mathrm{a}+1$ with a sub-path that ends with -1 .

Lemma 5. Let $H=(V, E)$ be a 3-hypergraph, $C$ a Hamiltonian cycle of $I_{2}(H)$ and $l$ an alternating labelling of $C$. If $v_{1}$ and $v_{2}$ are the only nodes of $H$ with signed degree different from zero with respect to $l$, say $d_{l}(u)=+2 k$ and $d_{l}(v)=$ $-2 k$, where $k \geq 1$, then $H$ admits a null labelling.

Proof. This is obtained by $k$ successive runs of Balance $\left(u, v, l^{i}(C)\right)$, with $0 \leq$ $i<k$, where $l^{i+1}(C)$ is the labelling obtained as output of Balance $\left(u, v, l^{i}(C)\right)$. 
We set $l^{0}=l$; the output $l^{k}$ of $\operatorname{Balance}\left(u, v, l^{k-1}(C)\right)$ provides a null labelling of $H$.

We emphasize that, from the second run of Balance() until the last one, i.e., the $k$-th run, the choice of a new starting sub-path $p_{u}$ is always possible. In fact, from $d_{l}(u)=+2 k$ it follows that in $l(C)$ the number of sub-paths of $u$ whose labels sum up to +1 exceeds exactly by $k$ those whose labels sum up to -1 . A last remark is required: since two different runs of Balance() start from different sub-paths $p_{u}$, their computations do not involve the same sub-path twice. So, each call $S$ witch() in the $k$ runs of Balance() always modifies two elements of $C$ whose labels are alternate, as set by $l(C)$.

This same reasoning can be generalized when more than two vertices of $H$ have non null signed degree leading to our main result

Theorem 2. Let $H$ be a 3-hypergraph. If the 2-intersection graph $I_{2}(H)$ is Hamiltonian, then $H$ admits a null labelling.

The proof of this theorem and the related computation of the null label of $H$ directly follow from the proofs of Lemmas 4 and 5 , after observing that we can iterate the calls of Balance $(u, v, l(C))$ varying $u$ among all the vertices with signed degree greater than zero until reaching the first vertex $v$ among those having signed degree less than zero. The following example will clarify the situation.

Example 4. Consider the following 3-hypergraph $H=(V, E)$ with $V=$ $\{1, \ldots, 8\}$ and $\mathrm{E}=\{\{2,3,5\},\{2,5,8\},\{2,4,8\},\{1,4,8\},\{1,4,7\},\{1,6,7\},\{1,4,6\}$, $\{1,5,6\},\{5,6,7\},\{1,5,7\},\{1,2,7\},\{1,2,3\},\{2,3,6\},\{3,6,8\},\{3,7,8\},\{3,5,8\}\}$

Figure 5 shows a Hamiltonian cycle $C$ of $I_{2}(H)$, and one of its alternating labellings $l(C)$.

The chosen labelling is not a null labelling of $H$. The vector of the signed degrees of the vertices of $H$ is

$$
d=(-2,2,0,2,-2,0,2,-2) .
$$

Let us perform a sequence of runs of Balance() to compute a null labelling of $H$ starting from $l(C)$.

Let us start, as an example, the run $\operatorname{Balance}(2, v, l(C))$ in the $p_{2}$ sub-path having $\{2,3,5\}$ as first element. It calls $\operatorname{Switch}\left(p_{2}, p_{1}\right)$, with $p_{1}=\operatorname{next}\left(p_{2}\right)$ and $\left|p_{1}\right|$ even. Since $d_{l}(1)=-2$, we perform the choice $v=1$, and the switchings of $\{2,4,8\}$ and $\{1,4,8\}$ leading to the labelling $l^{1}(C)$ such that $d_{l^{1}}(1)=d_{l^{1}}(2)=0$, leaving the remaining labels unchanged.

Let us now arbitrarily choose the vertex 7 such that $d_{l^{1}}(7)=+2$ and run Balance $\left(7, v, l^{1}(C)\right)$ with the starting $p_{7}$ sub-path whose first element is $\{5,6,7\}$. The sub-path $p_{3}=\operatorname{next}\left(p_{7}\right)$ has odd length so the labels of $\{1,2,7\}$ and $\{1,2,3\}$ are switched and we obtain $d(7)=0$ and $d(3)=+2$. Now $p_{8}=\operatorname{next}\left(p_{3}\right)$ and the labels of $\{2,3,5\}$ and $\{2,5,8\}$ are switched obtaining $d(3)=d(8)=0$. Since $\left|p_{8}\right|$ is even, the run Balance $\left(7, v, l^{1}(C)\right)$ ends setting $v=8$. A new labelling $l^{2}(C)$ is returned as output. 


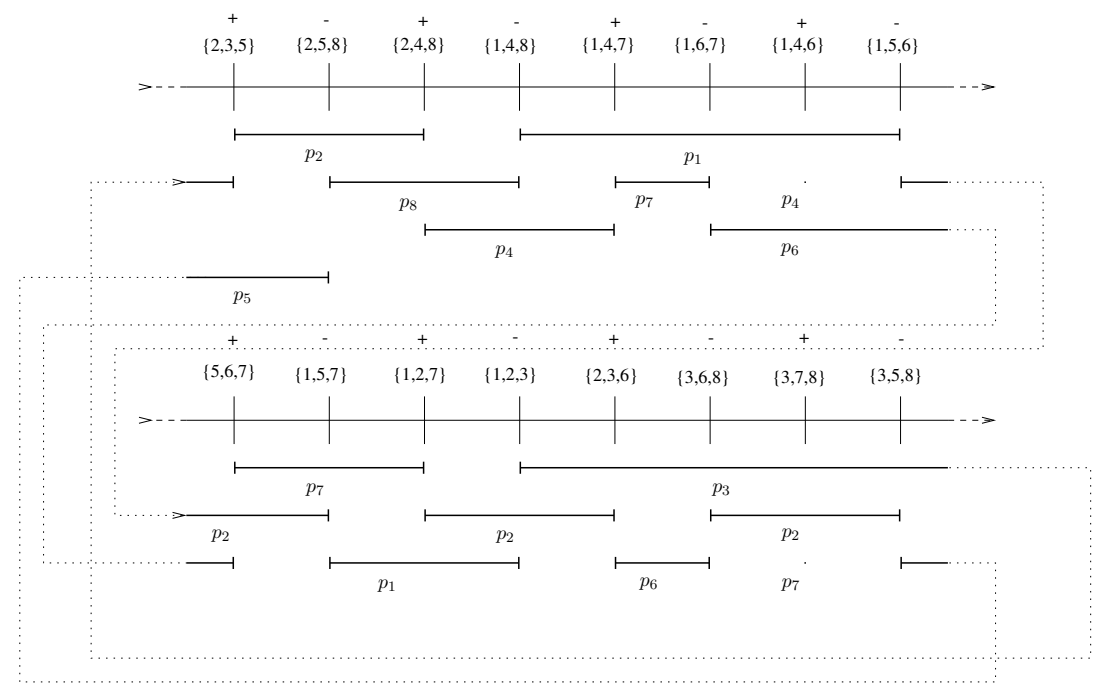

Fig. 5. A Hamiltonian cycle of $I_{2}(H)$ and its labelling.

Two more vertices with signed degree different from zero are left, i.e., the vertices 4 and 5. A last run of Balance $\left(4,5, l^{2}(C)\right)$ is performed. Taking the $p_{4}$ subpath containing only $\{1,4,6\}$, we have $p_{5}=\operatorname{next}\left(p_{4}\right)$ with $\left|p_{5}\right|$ even. Therefore, switching the sign of $\{1,4,6\}$ and $\{1,5,6\}$ we obtain a new labelling $l^{3}$ such that $d_{l^{3}}(4)=d_{l^{3}}(5)=0$ and Balance $\left(4,5, l^{2}(C)\right)$ ends. Therefore, the labelling

$$
l^{3}=(-1,1,-1,1,1,-1,-1,1,1,-1,-1,1,1,-1,1,-1)
$$

is a null labelling of $H$. Note that the order of the calls of Balance() is not relevant in order to obtain a null labelling of $H$.

\section{References}

1. Behrens, S., Erbes, C., Ferrara, M., Hartke, S.G., Reiniger, B., Spinoza, H., Tomlinson, C.:New Results on Degree Sequences of Uniform Hypergraphs, Electron. J. Comb., 20(4), \# P14 (2013).

2. Berge, C.: Hypergraphs, North-Holland, Amsterdam, (1989).

3. Brlek, S., Frosini, A.: A Tomographical Interpretation of a Sufficient Condition for h-Graphical Sequences DGCI 2016, Lect. Notes in Comput. Sc., 9647, pp. $95-104$ (2016).

4. Colbourne, C.J., Kocay, W.L. , Stinson, D.R.: Some NP-complete problems for hypergraph degree sequences Discrete Appl. Math., 14, pp. 239 - 254 (1986).

5. Dewdney, A.K., Degree sequences in complexes and hypergraphs, Proc. Amer. Math. Soc., 53(2), pp. $535-540$ (1975).

6. Deza, A., Levin, A., Meesum, S.M., Onn, S.: Optimization over degree sequences, SIAM J. Disc. Math. 32(3), pp. $2067-2079$ (2018). 
7. Erdös, P., Gallai, T.: Graphs with prescribed degrees of vertices (Hungarian), Math. Lapok. 11 pp. $264-274$ (1960).

8. A. Frosini, W.L. Kocay, G. Palma, L. Tarsissi, On null 3-hypergraphs, Discrete Appl. Math. in press (2021) https://doi.org/10.1016/j.dam.2020.10.020.

9. A. Frosini, C. Picouleau, S. Rinaldi, On the degree sequences of uniform hypergraphs Lect. Notes in Comput. Sc. 7749 (2013) 300-311.

10. A. Frosini, C. Picouleau, S. Rinaldi, New sufficient conditions on the degree sequences of uniform hypergraphs Theoret. Comput. Sc. (2021) in press.

11. Harary, F.: Graph Theory, Addison Wesley Publishing Company (1972).

12. Herman, G.T., Kuba, A. (Eds.): Discrete tomography: Foundations algorithms and applications, Birkhauser, Boston (1999).

13. Herman, G.T., Kuba, A. (Eds.): Advances in Discrete Tomography and Its Applications (Applied and Numerical Harmonic Analysis), Birkhauser, Boston (2007).

14. Kocay, W., Li, P.C.: On 3-Hypergraphs with Equal Degree Sequences, Ars Combinatoria, 82, pp.145 - 157 (2006)

15. Kocay, W.: A Note on Non-reconstructible 3-Hypergraphs, Graphs and Combinatorics 32, pp. 1945 - 1963 (2016) 